\title{
FORMULATION, DEVELOPMENT AND CHARACTERIZATION OF DRUG DELIVERY SYSTEMS BASED TELMISARTAN ENCAPSULATED IN SILK FIBROIN NANOSPHERE'S
}

\author{
SHAHID UD DIN WANI*, GANGADHARAPPA H. V., ASHISH N. P.
}

Department of Pharmaceutics, JSS College of Pharmacy, JSS Academy of Higher Education and Research, Sri Shivarathreeshwara Nagara Mysuru, India 570015

Email: shahidpharma2013@gmail.com

Received: 31 Oct 2018, Revised and Accepted: 12 Dec 2018

\section{ABSTRACT}

Objective: The aim of the present work was to formulate silk fibroin (SF) nanospheres (NS's) for drug delivery application. The current study was designed to advance the water solubility and bio-availability of telmisartan by nanoprecipitation method.

Methods: SF NS's loaded with TS were prepared by nanoprecipitation method. The drug was dissolved in aqueous solution of SF by using acetone as a non-solvent. The prepared NS's were then characterized by FTIR, X-ray diffraction and zeta potential, and were evaluated for its, surface morphology, \%drug content, encapsulation efficiency and in vitro drug release.

Results: The evaluation results of SF NS's loaded of TS showed 74.22 $\pm 0.17 \%$ entrapment efficiency, $35.21 \pm 0.02 \%$ of drug loading, and- $4.9 \mathrm{mV}$ to$13.6 \mathrm{mV}$ of zeta potential due to the proper bounding of TS with the $\beta$-sheets of SF, the particle size reported was within the size range of 160-186 $\mathrm{nm}$ having smooth surface and were spherical in shape. The SFNS's pattern switched from random coil to $\beta$-sheet formation on treating with acetone. FTIR and DSC studies marked no such inter-molecular interactions between SF and drug molecules. The \% cumulative in vitro drug release from SF NS's exhibited quick burst release. The in vitro cumulative drug release of SF NS's of TS it was found that about $74 \%$ of the drug was released within $8 \mathrm{~h}$ and about $96 \%$ of drug released at $24 \mathrm{hr}$. The rate of drug release increased with the increase in SF ratio.

Conclusion: It is believed that these SF NS's will find potential applications in drug delivery release as drug carriers, especially poor water-soluble drugs. All these results proposed that SF NS's are eventuality handy in various drug delivery systems.

Keywords: Bombyx mori, Silk Fibroin, Telmisartan, Nanospheres, Drug delivery

(C) 2019 The Authors. Published by Innovare Academic Sciences Pvt Ltd. This is an open access article under the CC BY license (http://creativecommons.org/licenses/by/4.0/) DOI: http://dx.doi.org/10.22159/ijap.2019v11i1.30588

\section{INTRODUCTION}

Silk fibroin (SF) obtained from Bombyx mori silk cocoons are one of the proteins which have been extensively explored in the biomedical and pharmaceutical domain [1, 2]. Some researchers have reported various processes to develop the SF nanospheres by self-build of SF macromolecules. Researchers have found that Regenerated-SF and poly-(vinyl alcohol) PVA were macroscopically or microscopically separated into various stages in SF/poly-(vinyl alcohol) blend films. SF microspheres or nanospheres have been attained by establishing the blend film into $\mathrm{H}_{2} \mathrm{O}$ to diffuse the poly (vinyl alcohol) factor [35]. Zhang et al., 2007 formed regenerated-SF nanospheres by the simple addition of regenerated-SF solution into the $\mathrm{H}_{2} \mathrm{O}$-miscible polar organic solvents, such as $\mathrm{C}_{2} \mathrm{H}_{5} \mathrm{OH}, \mathrm{CH}_{3} \mathrm{OH}, \mathrm{n}-\mathrm{C}_{3} \mathrm{H}_{7} \mathrm{OH}, \mathrm{C}_{3} \mathrm{H}_{8} \mathrm{O}$, $\mathrm{C}_{4} \mathrm{H}_{8} \mathrm{O}$, and $\mathrm{C}_{3} \mathrm{H}_{6} \mathrm{O}$ [6]. Regenerated Silk Fibroin (RSF) devices, for e. g., fibers, nanospheres, films and particles have been reported for various applications [7]. Due to their degradation properties biodegradable polymeric nanospheres have been often proposed to be used in controlled release drug delivery systems [8,9]. Telmisartan (TS) is an anti-hypertensive drug. It is an angiotensin-II receptor antagonist. It acts by the binding to the angiotensin-II type-I receptors, ensuing in the inhibition of angiotensin-II on vascular smooth muscles. As angiotensin-II is a vasoconstrictor [10], inhibition of its effect on vascular smooth muscles [11] results in decreases in systemic vascular resistance [12]. TS come under the class-II of BCS Class. Essentially, as a BCS class-II drug, TS is very poorly soluble in $\mathrm{H}_{2} \mathrm{O}$, which results in the reduced dissolution and accordingly lower bioavailability when dispensed orally [13-15, 3536]. By using the hydrophobic carriers to overcome the lower bioavailability, $\mathrm{SF}$ as a carrier can be used to increase the bioavailability of TS. Furthermore, $\mathrm{C}_{3} \mathrm{H}_{6} \mathrm{O}$ functioned both as the solvent of TS and the additive for NS formulation, which assembled the procedure easy and further productive.

SF obtained from Bombyx mori silk, a type of natural protein polymer with very well bio-compatibility and biodegradability [1], and has newly been broadly explored as a bio-material, such as a matrix for cell-based culture substrates [16] and drug delivery systems [17]. These objectives, SF based materials such as films [18] microparticles [5] and nanoparticles [6] nanospheres [19], had been prepared and described. The minimum inflammatory reactions in vitro and in vivo of SF matrices have also been described by [20].

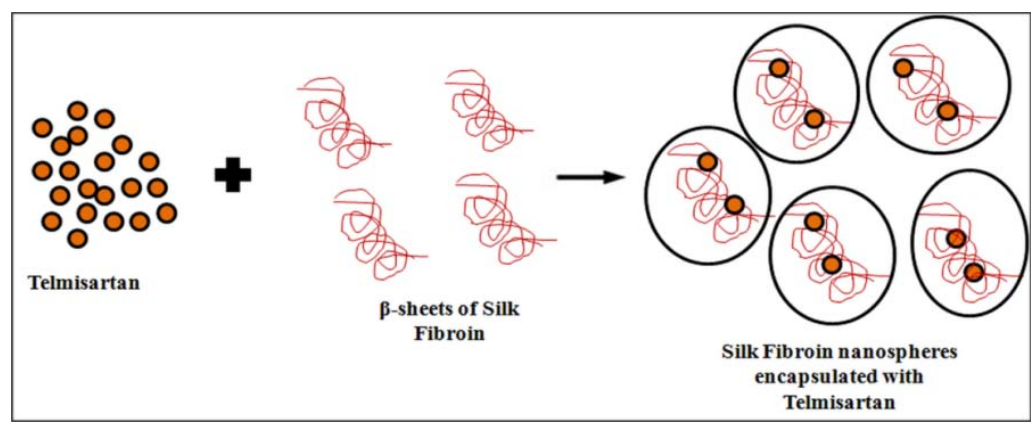

Schema 1: Depiction of telmisartan encapsulating with silk fibroin nanospheres 
In the present work, we developed a novel and simplistic procedure to formulate SF nanospheres for drug delivery application. Telmisartan-silk fibroin nanospheres (TSSF-NS) were produced by amassing of silk fibroin protein in aq. sol. at RT devoid of casting any harmful organic solvents, surfactants or other toxin ingredients. The current study was designed to advance the water solubility and bioavailability of telmisartan by nanoprecipitation method. The developed SFNS as a carrier for TS, the hydrophobic core of SFNS yields the right domain to encapsulate TS by hydrophobic interactions, with that nanospheres showed TS got attached with the $\beta$-sheets form of SF as shown in (schema 1).

In addition, we also explored the influence of different ratios of silk fibroin with regards to drug release. The investigation further figured the encapsulated telmisartan nanospheres (TSSF-NSs), for encapsulation, drug loading, particle size, surface properties, their morphology of SFN s's and it's in vitro drug release and release kinetics to explore its use as a potential drug delivery carrier.

\section{MATERIALS AND METHODS}

\section{Materials}

Bombyx mori Silk Cocoons were provided by Central Sericultural Research and Training Institute, Mysuru, India. Telmisartan was gifted by Vowcare Pharma, JandK. A. R. grade Sodium Carbonate $\left(\mathrm{Na}_{2} \mathrm{CO}_{3}\right)$ and Acetone $\left(\mathrm{C}_{3} \mathrm{H}_{6} \mathrm{O}\right)$. were obtained from Loba Chemie Pvt. Ltd., India. Lithium Bromide was obtained from Axiom Chemicals, Gujarat, India. dd- $\mathrm{H}_{2} \mathrm{O}$ was obtained from Nice Chemicals, Kerala, India. All reagents were of A. R. grade.

\section{Methods}

\section{Preparation of silk fibroin solution}

As described in earlier methods [21], Bombyx mori silk cocoons were cropped into little slices and heated for about $45 \mathrm{~min}$ in aq. sol. of $0.02 \mathrm{M} \mathrm{Na} \mathrm{Na}_{3}$, and then washed meticulously with $\mathrm{dd}-\mathrm{H}_{2} \mathrm{O}$. Afterward desiccating at RT, the de-gummed silk was mixed in $9.3 \mathrm{M}$ of aq., Lithium Bromide solution. After dialysing against $\mathrm{dd}-\mathrm{H}_{2} \mathrm{O}$ for $96 \mathrm{~h}$ at RT by using $3.5 \mathrm{kDa}$ cut off dialysis cassette, the silk fibroin (SF) aq., the solution was centrifuged at $9000 \mathrm{rpm}$ per min for 15 min to detach traces of fibres. The conc. of SF sol. was measured by weighting technique. Lastly, the SF sol. was diluted with a certain conc of dd- $\mathrm{H}_{2} \mathrm{O}$ water and stored at $2-4{ }^{\circ} \mathrm{C}$ for further use.

\section{Preparation of drug-loaded silk fibroin nanospheres}

Telmisartan (TS) loaded silk fibroin (SF) nanospheres (NS's) were obtained by a nanoprecipitation technique in acetone (Zhang et al., 2007) [6]. The nature of the method was elucidated as follows. An appropriate amount of drug was dissolved in $2.5 \mathrm{ml}$ of SF solution with drug: SF ratios of $1: 1,1: 2$ and $1: 3$ and $1: 4(\mathrm{w} / \mathrm{w})$. The solution was poured into $10 \mathrm{ml}$ of acetone with stirring. The suspensions of TSloaded nanospheres were maintained for $2 \mathrm{~h}$. Then the TS-loaded nanospheres were recovered by centrifugation at 9,500 rpm for 15 min to separate these nanospheres from acetone medium before the freeze-drying. The SFNS's without drug loading was also prepared as a control.

\section{Determination of \% yield, entrapment efficiency, and drug loading}

Determination of \% yield of nanospheres of telmisartan (TS) was determined by the following formula

$$
\text { (\%) Yield }=\frac{\text { Practical yield }}{\text { Theoretical yield }} \times 100
$$

The prepared nanospheres of TS were centrifuged with $10 \mathrm{ml}$ of methanol at $4500 \mathrm{rpm}$ for $15 \mathrm{~min}$. The free amount of the TS loaded NS's in the filtrate was determined by UV-Vis spectroscopy at 270 $\mathrm{nm}$ respectively. Measurements were observed in triplicate. The entrapment efficiency (EE) and drug loading (DL) were calculated by the following formula [18]:

$$
\mathrm{EE}=\frac{\text { Tot. Amt. of drug- Amt. of free drug }}{\text { Tot. Amt. of drug }} \times 100
$$

$$
\mathrm{DL}=\frac{\text { Wt. of the drug drug }}{\text { Wt. of the nanospheres }} \times 100
$$

Determination of particle size (PS) and zeta potential (ZP) and stability studies of silk telmisartan loaded fibroin nanospheres

The mean dia of TS loaded nanospheres were measured by laser diffraction using a NANOTRAC WAVE II instrument (Microtrac, U. S. A.) at a determined scattering angle of $90^{\circ}$ at $25^{\circ} \mathrm{C}$. Zeta potential is a commonly used the parameter to characterize the surface charge property and stability of nanospheres. The SFNS loaded with Telmisartan, were diluted with $\mathrm{dd}-\mathrm{H}_{2} \mathrm{O}$ and PS and ZP were determined [22].

The stability of prepared nanosphere was determined [23], by monitoring their mean particle size and zeta potential in de-ionized water using DLS particle size analyzer at a fixed angle of $90^{\circ}$ at 27 ${ }^{\circ} \mathrm{C}$. After preparation, the nanospheres were incubated at $37^{\circ} \mathrm{C}$ in the incubator, and their sizes were determined after 1, 6, 12, 24, 48, 96 , and $168 \mathrm{~h}$ respectively.

\section{FTIR analysis}

Functional groups of SF and drug (TS) of the NS's and interactions $\mathrm{b} / \mathrm{w}$ them were studied by FT-IR spectroscopy using FTIRspectrophotometer (Shimadzu-8400, Japan), taking air as a reference. The resolution of $4 \mathrm{~cm}^{-1}$ and 32 scans were used. FT-IR spectrum was obtained from a KBr disk of dried NS's [24].

\section{DSC studies}

Thermal properties of the NS's were determined by thermogravimetry (TG) by using TA-Instrument (Shimadzu DSC-60, Japan), TGA. For TG analysis, sample $(10 \mathrm{mg})$ was heated from $50^{\circ}$ to $350{ }^{\circ} \mathrm{C}$ at a heating rate of $20^{\circ} \mathrm{C} / \mathrm{min}$ under nitrogen atmosphere.

\section{X-Ray diffraction studies}

XRD patterns of pure TS and TS-SFNS5 were attained using a powder X-ray diffract meter (Rigaku Ultima III), Rigaku Corporation, Japan. The samples were studied by locating a thin sheet of powder in the traditional cavity frames. The scanning rate was $2.5 \% \mathrm{~min}$ at a diffracting angle $(2 \theta)$ of 0 to $45^{\circ} \mathrm{C}$.

\section{SEM analysis}

Morphologies of nanospheres were investigated by scanning electron microscopy (SEM) using a Hitachi NORAN System 7 (Japan). The nanospheres were coated with Au for improving conductivity before the scan. The avg. sizes of nanospheres were determined from various SEM images counting a least of 100 particles by using smile view software (version 1.02).

\section{In vitro drug release}

Specific amt (about $5 \mathrm{mg}$ ) of TS-loaded NS was placed into a dialysis membrane tube containing $3.0 \mathrm{ml}$ of Phosphate Buffer Solution (PBS) having a $\mathrm{pH}$ of 7.4. The sampling tubes were enshrouded at 37 ${ }^{\circ} \mathrm{C}$ at the shaking rate of $120 \mathrm{rpm}$ [25-27]. At changed time intervals, the tubes were centrifuged at 9,500 rpm for $10 \mathrm{~min}$ before separating the PBS medium. After that freshly prepared $3 \mathrm{ml}$ of PBS was added into the tube for continuing release test. UV-Vis spectrophotometer was used to measure the released telmisartan. As stated, to a different telmisartan conc. in absorbance standard curve, telmisartan conc. of the release, medium was observed and \% telmisartan release was calculated. Each avg. the value was cal. from the three measurements.

\section{Release Kinetics}

Data obtained from in vitro analysis were adapted to different kinetic equations to establish the method of drug release from SF nanospheres. The kinetic equations used were zero-order equation, 1 st-order equation and Higuchi-model [28].

\section{$\mathrm{C}=\mathrm{k}_{0} \mathrm{t}$}

$\mathrm{k}_{0}$ is zero order rate constant defined in units of conc./ $\mathrm{t}$ and $(\mathrm{t})$ time. $\log C=\log _{0}-k_{1} t / 2.303$ 
Where $\mathrm{C}_{0}$ is the incipient conc. of drug and $\mathrm{k}_{1}$ is 1 st-order constant. $\mathrm{Q}=\mathrm{k}_{\mathrm{H}} \mathrm{t}^{1 / 2}$

Where $\left(\mathrm{k}_{\mathrm{H}}\right)$, is Higuchi constant musing scheme parameters of the system.

The rate constants were designed for the individual models. Additional, to locate out the process of drug release, the first $50 \%$ drug release was ensuite in Korsmeyer-Peppas model [30]:

\section{$\mathrm{Q}_{\mathrm{t}}=\mathrm{k} \mathrm{t}^{\mathrm{n}}$}

Where $\mathrm{k}$ is the kinetic-constant and $\mathrm{n}$ is the diffusion-exponent, an evaluation of the $1^{\circ}$ means of the drug release.

\section{Statistical analysis}

All reports were listed as mean \pm sem. Statistical analyses of reports were made by ANOVA or Student's t-test. It was assessed considerably distinct if $\mathrm{p}<0.05$.

\section{RESULT AND DISCUSSION}

\section{Evaluation of telmisartan loaded-silk fiboin nanosphere's}

The prepared nanospheres were evaluated for physical appearance, $\%$ yield, entrapment efficiency (EE), drug loading (DL), particle size (PS) and zeta potential (ろ). The physical appearance of the silk fibroin nanospheres loaded with telmisartan was found to be yellow whitish colour powder and odourless. The silk fibroin loaded nanospheres of telmisartan were prepared by the aqueous solvent method as mentioned in (table 1); also earlier methods used by [34] in their studies, methanol (or ethanol) was commonly used the method to increase $\beta$-sheet content. In the present study, acetone containing pure telmisartan (TS) was directly added to the silk fibroin solution so as to increase the drug loading and drug release.

\section{Entrapment efficiency and drug loading}

The evaluation results of silk fibroin nanosphere formulations (TSSF-NS1 to TSSF-NS5) of telmisartan showed lowest $42.18 \pm 0.23$ $\%$ and highest $58.13 \pm 0.21 \%$ yield respectively given in (table 2 ).

Table 1: Composition of telmisartan loaded silk fibroin nanospheres

\begin{tabular}{lllll}
\hline S. No. & Formulation code & \multicolumn{2}{c}{ Ratios } & Scetone \\
\cline { 3 - 5 } & & Telmisartan & 1 & 0.5 \\
\hline 01 & TSSF-NS1 & 0 & 1 & 0.5 \\
02 & TSSF-NS2 & 1 & 2 & 0.5 \\
03 & TSSF-NS3 & 1 & 3 & 0.5 \\
04 & TSSF-NS4 & 1 & 4 & 0.5 \\
05 & TSSF-NS5 & 1 & 4 & \\
\hline
\end{tabular}

\#TS-SFNS1 (Blank nanosphere)

Table 2: Evaluation of formulation batches of silk fibroin nanospheres of telmisartan mean particles size, entrapment efficiency, and drug loading

\begin{tabular}{|c|c|c|c|c|c|}
\hline \multirow[t]{2}{*}{ S. No. } & \multirow[t]{2}{*}{ Formulations } & \multicolumn{4}{|c|}{ Evaluation parameters } \\
\hline & & Yielda (\%) & Entrapment efficiency ${ }^{a}(\%)$ & Drug loadinga (\%) & Particle size a (nm) \\
\hline 01 & TSSF-NS1 & $29.52 \pm 0.20$ & $00.00 \pm 0.00$ & $00.00 \pm 0.00$ & $152.10 \pm 2$ \\
\hline 02 & TSSF-NS2 & $42.18 \pm 0.23$ & $52.22 \pm 0.11$ & $22.14 \pm 0.06$ & $160.12 \pm 3$ \\
\hline 03 & TSSF-NS3 & $53.22 \pm 0.14$ & $59.26 \pm 0.36$ & $27.22 \pm 0.11$ & $168.22 \pm 2$ \\
\hline 04 & TSSF-NS4 & $46.29 \pm 0.04$ & $66.17 \pm 0.15$ & $31.04 \pm 0.34$ & $174.09 \pm 4$ \\
\hline 05 & TSSF-NS5 & $58.13 \pm 0.21$ & $74.22 \pm 0.17$ & $35.21 \pm 0.02$ & $186.11 \pm 1$ \\
\hline
\end{tabular}

adata are given in mean $\pm \mathrm{SD}, \mathrm{n}=3, \mathrm{~nm}=$ nanometer

The entrapment efficiency and drug loading of silk fibroin nanospheres of telmisartan varies from $52.22 \pm 0.11$ to $74.22 \pm 0.17 \%$ and $22.14 \pm 0.06$ to $35.21 \pm 0.02 \%$ respectively. It was found that silk fibroin nanospheres of telmisartan TSSF-NS5 showed highest 74.22 $\pm 0.17 \%$ entrapment efficiency and $35.21 \pm 0.02 \%$ drug loading due to the proper bounding of telmisartan with the $\beta$-sheets of silk fibroin.

\section{Particle size, zeta potential, and Stability of nanospheres}

We first experienced the result of varying polymer concentrations by dilution on particle sizes. In this experiment, the weight ratio of silk fibroin solution was increased gradually from $1 / 1$ to $1 / 4$, while keeping the acetone in solution at constant. The particle size varies from $152.10 \pm 2 \mathrm{~nm}$ to $186.11 \pm 1 \mathrm{~nm}$ with (fig. 1) and without drug.

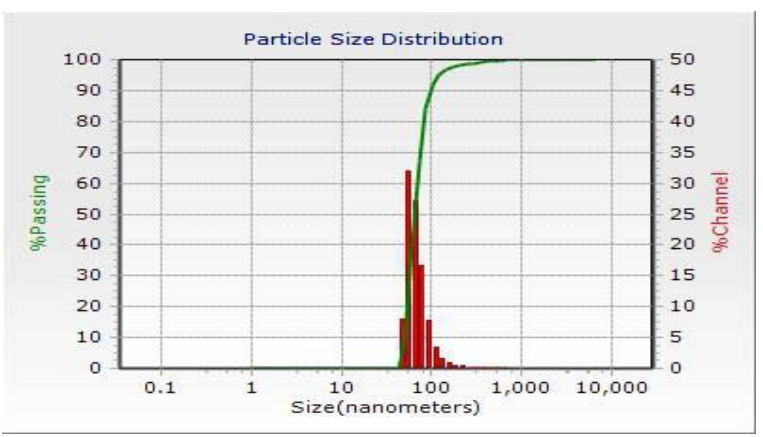

Fig. 1: Particle size of blank silk fibroin nanosphere
Cumulative analysis of particle size analysis records collected from the $1 / 1$ sample showed a relatively lower size allocation with a mean sphere size of $160.12 \mathrm{~nm} \pm 3$. The $1: 2$ and 1:4 samples, though, have bigger mean sizes (168.22 \pm 2 and $186.11 \pm 1$, respectively) and a wide sphere size division ranging from $150 \mathrm{~nm}$ to $200 \mathrm{~nm}$.

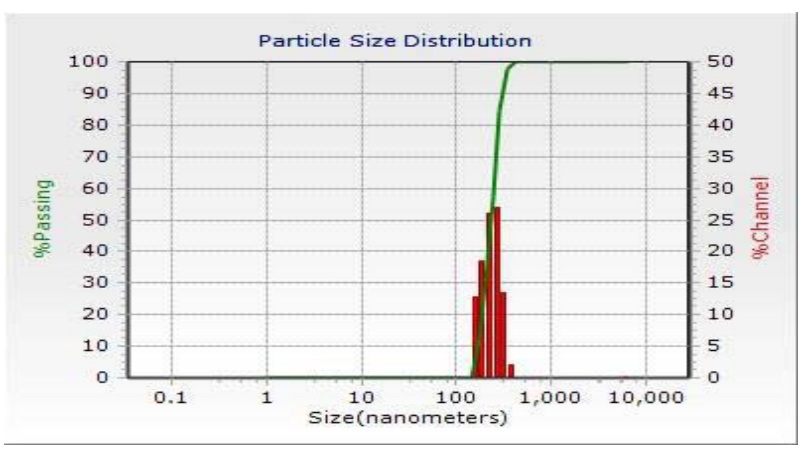

Fig. 2: Particle size of silk fibroin nanosphere loaded of telmisartan (TSSF-NS5)

In consideration of information in the literature on silk fibroin nanosphere's [30], using organic solvents with diverse molecular weights can as well modify mean sphere size and its size distribution. Although to reduce particle size we treated the above 
experiment with the constant volume of acetone. It was found that optimized silk fibroin nanosphere of telmisartan (TSSF-NS5) showed particle size of $186.11 \pm 1 \mathrm{~nm}$ (fig. 2) particle size due to the availability of numbers of TS molecule and combined interaction with $\beta$-sheets of silk fibroin.

The silk fibroin nanosphere loaded with telmisartan TS-SFNS5 with zeta potential above $\pm 10 \mathrm{mV}$ have been shown to be stable, as the surface charge prevents aggregation of the particles.

As shown in (table 3) and (fig. 3), the value of zeta potential (-13.3 $\mathrm{mV}$ ) showed that prepared silk fibroin nanoparticle with telmisartan TSSF-NS5 has sufficient charge and mobility to inhibit aggregation of particles.

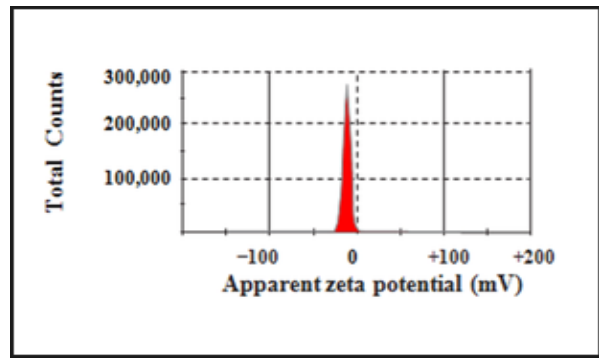

Fig. 3: Zeta-potential of silk fibroin nanosphere loaded with telmisartan (TSSF-NP5

Table 3: Evaluation of formulation batches of silk fibroin nanospheres of Telmisartan for Particle size and zeta potential

\begin{tabular}{llll}
\hline S. No. & Formulation code & Particle size (nm) $^{\mathbf{a}}$ & Zeta potential (ろ) $^{\mathbf{b}}$ \\
\hline 01 & TSSF-NS1 & $152.10 \pm 2$ & $-4.9 \mathrm{mV}$ \\
02 & TSSF-NS2 & $160.12 \pm 1$ & $-7.8 \mathrm{mV}$ \\
03 & TSSF-NS3 & $168.22 \pm 4$ & $-9.2 \mathrm{mV}$ \\
04 & TSSF-NS4 & $174.09 \pm 3$ & $-11.7 \mathrm{mV}$ \\
05 & TSSF-NS5 & $186.11 \pm 1$ & $-13.3 \mathrm{mV}$ \\
\hline
\end{tabular}

a,b data are given in mean $\pm S D, n=3, n m=$ nanometer

The constancy of the sphere suspension, as noticed during sample storage at $4{ }^{\circ} \mathrm{C}$ for up to $3 \mathrm{mo}$, ensued a comparable fashion to the particle size, with the $50 \%$ sonicated sample found to be more stable. Hence, silk fibroin loaded with telmisartan nanospheres has the potential of physical stability. These nanospheres were found to be stable in de-ionized water over a longer period of time (table 4).

Table 4: Determination of stability of telmisartan encapsulated silk fibroin nanospheres using the particle size analyzer in de-ionized water

\begin{tabular}{|c|c|c|}
\hline \multicolumn{3}{|c|}{ De-ionized water } \\
\hline Time & Particle size (nm)a & 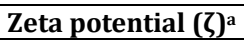 \\
\hline $1 \mathrm{~h}$ & $183.10 \pm 1$ & $-12.79 \mathrm{mV}$ \\
\hline $6 \mathrm{~h}$ & $181.12 \pm 3$ & $-11.88 \mathrm{mV}$ \\
\hline $12 \mathrm{~h}$ & $186.22 \pm 1$ & $-13.30 \mathrm{mV}$ \\
\hline $48 \mathrm{~h}$ & $186.09 \pm 4$ & $-13.11 \mathrm{mV}$ \\
\hline $96 \mathrm{~h}$ & $182.09 \pm 2$ & $-12.39 \mathrm{mV}$ \\
\hline $168 \mathrm{~h}$ & $184.09 \pm 3$ & $-12.88 \mathrm{mV}$ \\
\hline
\end{tabular}

adata are given in mean $\pm \mathrm{SD}, \mathrm{n}=3$

\section{FTIR analysis}

The absorption bands in the frequency range of 1539-1623 $\mathrm{cm}^{-1}$ were ascribed to the random coil of SF, 1271 and $1623 \mathrm{~cm}^{-1}$ ascribed to $\beta$-sheets. To ascertain the $\beta$-sheet content, FTIR was accomplished on silk fibroin loaded telmisartan nanospheres. In earlier studies with silk films/nanosphere, it has been established that the nanosphere's originally demonstrated mostly amorphous structure
(1532 $\left.\mathrm{cm}^{-1}\right)$ with a few silk $I$ arrangement (1652 and $\left.1655 \mathrm{~cm}^{-1}\right)$ [31]. Following dealing with acetone, the silk $I$ structure was predominant with the increased in the $\beta$-sheets formation of above $40 \% \beta$-sheets. FT-IR spectrum of TS displays distinctive peaks at $3443 \mathrm{~cm}^{-1}$ (N-H stretch), $2959 \mathrm{~cm}^{-1}$ (aromatic C-H stretch), 2926 $\mathrm{cm}^{-1}$ (aliphatic C-H stretch), $1698 \mathrm{~cm}^{-1}$ (carbonyl group), $1453 \mathrm{~cm}^{-1}$ express the existence of $\mathrm{C}=\mathrm{C}$ aromatic group and the peak $1267 \mathrm{~cm}^{-1}$ for $\mathrm{O}-\mathrm{H}$ stretching vibration as shown in (fig. 4).

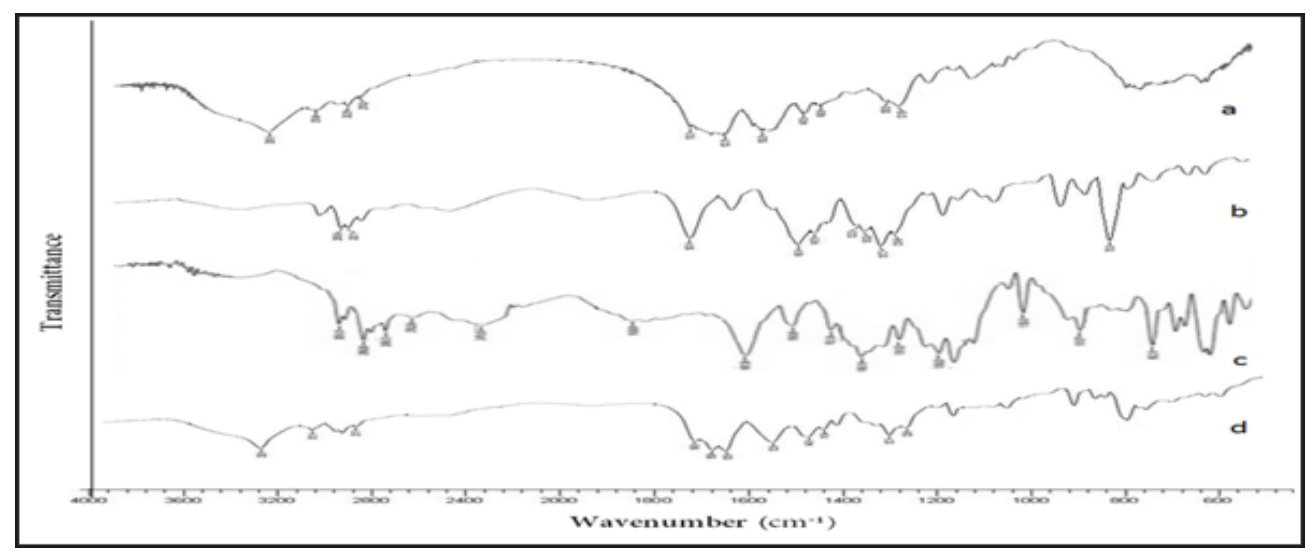

Fig. 4: (a) FTIR of Pure drug telmisartan, (b) FTIR of silk fibroin, (c) FTIR physical mixture of pure drug and polymer, and (d) FTIR of telmisartan nanosphere TSSF-NS5 by using FTIR-spectrophotometer shimadzu-8400 (scan range from 600-4000 $\mathrm{cm}^{-1}$ ) 
FT IR spectrum of silk fibroin loaded telmisartan nanospheres (TSSF-NS5) also shows distinctive peaks at $3280 \mathrm{~cm}^{-1}$ (N-H stretch), $3061 \mathrm{~cm}^{-1}$ (aromatic C-H stretch), $2870 \mathrm{~cm}^{-1}$ (aliphatic C-H stretch), $1694 \mathrm{~cm}^{-1}$ (carbonyl group), $1444 \mathrm{~cm}^{-1}$ signifies the aspect of $(\mathrm{C}=\mathrm{C}$ )aromatic group and the peak $1267 \mathrm{~cm}^{-1}$ for $0-\mathrm{H}$ stretching vibration. Presence of all these peaks and the paucity of some fresh peaks in the TSSF-NS5 formulation indicates no chemical communion amongst the drug and polymer.

\section{DSC studies}

The DSC thermogram of pure telmisartan demonstrated a sharp peak at $273^{\circ} \mathrm{C}$ (fig. 5), which coincides to the melting temperature of telmisartan, the sharpness of the peak of the drug demonstrating its crystalline nature and a single peak showed that the drug sample is free from any impurities.

The thermogram of silk fibroin showed a peak at $320^{\circ} \mathrm{C}$, which corresponds to its melting temperature. In the optimized formulation, telmisartan-loaded silk fibroin (TSSF-NS5) sharp drug: peaks were observed first at $268^{\circ} \mathrm{C}$ indicating that the crystallinity of the drug was reduced and it might be converted to amorphous form. Although typical endothermic peak similarly to the drug melting expanded and moved towards lower temp., with the lower intensity in SFNS's formulation. This might be ascribed to the shift of crystalline description of the drug in the TSSF-NS5.

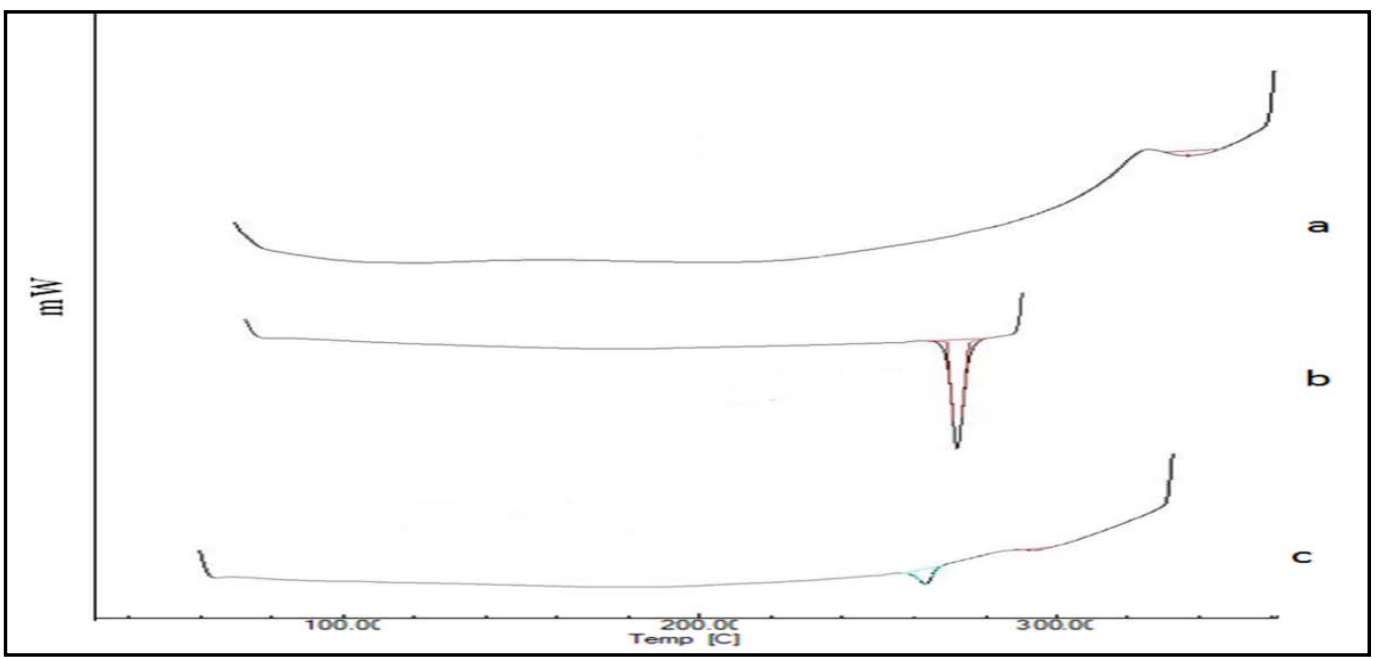

Fig. 5: (a) DSC of silk fibroin, (b) DSC of telmisartan, and (c) DSC of silk fibroin nanospheres TSSF-NS5

\section{XRD studies}

XRD arrangement of pure TS and TS-SFNS-5 is shown in (fig. 6). The diffraction pattern of TS asserted precisely the high-intensity peaks at diffraction angles $(2 \theta)$ of $6.3^{\circ}, 10.1^{\circ}, 15.53^{\circ}, 16.32^{\circ}, 17.5^{\circ}, 18.11$ $\circ 21.6^{\circ}, 23.6^{\circ}$, and $25.21^{\circ}$ implying that the drug endured as a crystalline material.
The XRD pattern of TS-SFNS- 5 exhibited an appreciable decline in the peak strength correlated with distinctive peaks of pure TS. This declined peak recommends modification of the drug chiefly into an amorphous shape. This distinct decline in peak strength furnishes an evidence for the important inflation in the disso-rates and, consequently bio-availability by SFNS formulation.

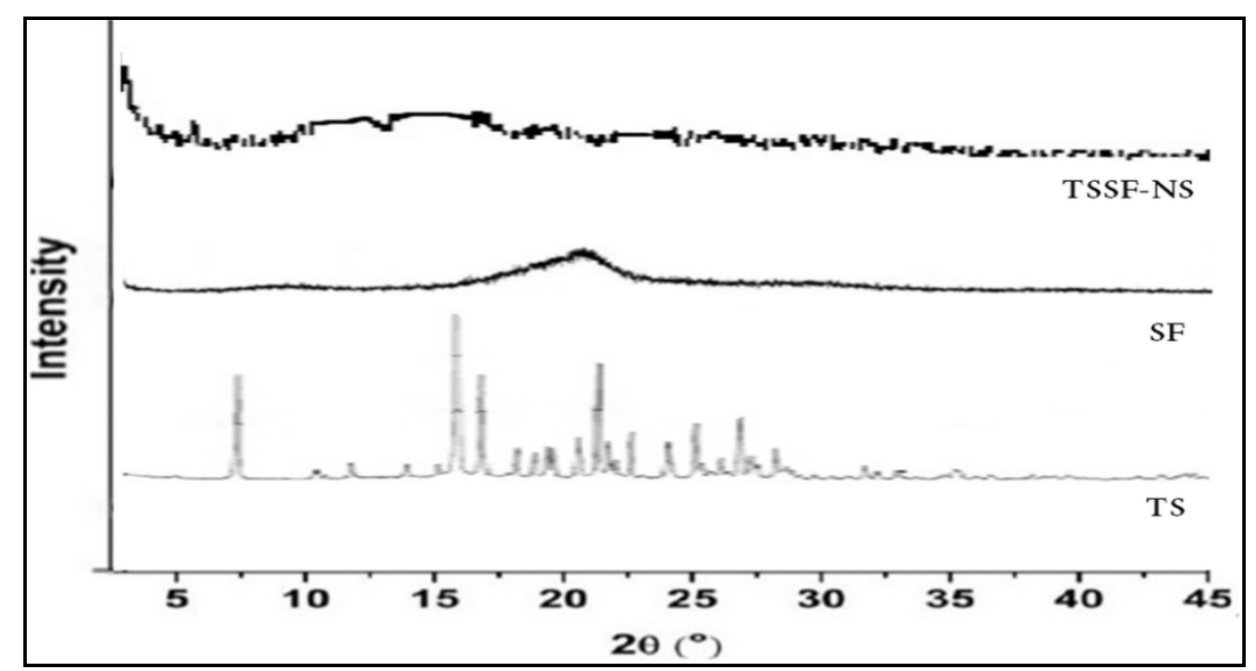

Fig. 6: (a) XRD of Telmisartan, (b) XRD of Silk Fibroin, and (c) XRD of Silk Fibroin nanospheres TSSF-NS5 using X-ray diffractometer (Rigaku Ultima III, Rigaku Corporation) $2 \theta$ range of $0^{\circ}$ to $45^{\circ} \mathrm{C}$

\section{SEM analysis}

The TS-loaded SFNS with bulbous in shape and had smooth surface can be prepared by the nanoprecipitation technique according to [7].
As shown in (fig. 7), the results of SEM analysis indicated that the different drug loading contents did not affect to morphology and size of SF nanospheres. 


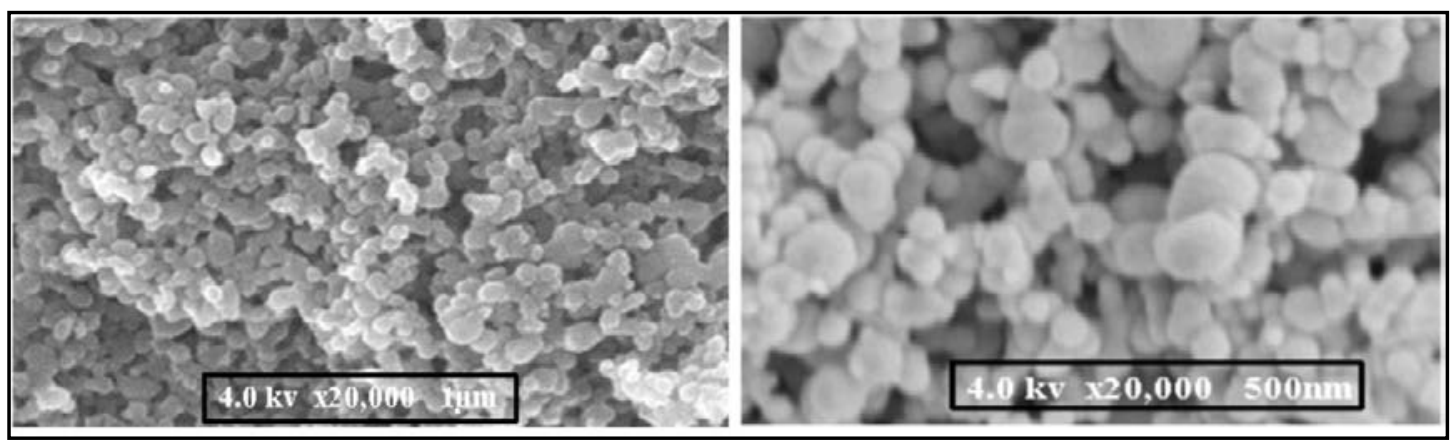

Fig. 7: SEM of silk fibroin nanospheres TSSF-NS5 using scanning electron microscope (Hitachi NORAN system 7)

It should be noted that the nanoparticles with a spherical shape called as nanospheres should give steadier drug release than the nanospheres with uneven contour.

\section{In vitro drug release study}

For the drug release behaviors, the drug release increased as the decreasing drug ratio. The reason for this could be that the SF nanospheres with higher drug content predominantly contained the $\beta$ sheet SF conformation as described in FTIR analysis. The drug was released from the higher content of $\beta$-sheet $\mathrm{SF}$ nanosphere matrix. Hence, related to phospholipids, the amphiphilic character of SF molecules facilitates the loading of both hydrophobic and hydrophilic drugs in SF NS's owing to its inter-molecular interactions, with the possibility to manage drug loading and release by altering the degree of
SF crystallinity ( $\beta$-sheet) structure arrangement. As compared with extensively used lipid vesicles, due to their elevated molecular weight, amino acid composition, and unique structure, SF vesicles are more chemically and physically stable and more suitable for delivering macromolecular drugs. The SF NS's reported in the present study, based on the ability to control size and shape, can be incorporated in these systems and used as a carrier for drug delivery. Silk microspheres and nanospheres prepared with different methods have also been reported [32-34]. Compared to these methods, the present study furnishes a singular method that can manage the size and shape of the nanospheres in accordance with detailed conditions. The method compels no organic solvents and no pricey apparatus during substance dispensation; as a result, this will be appropriate for the potential bio-medical and pharmaceutical applications for drug delivery.

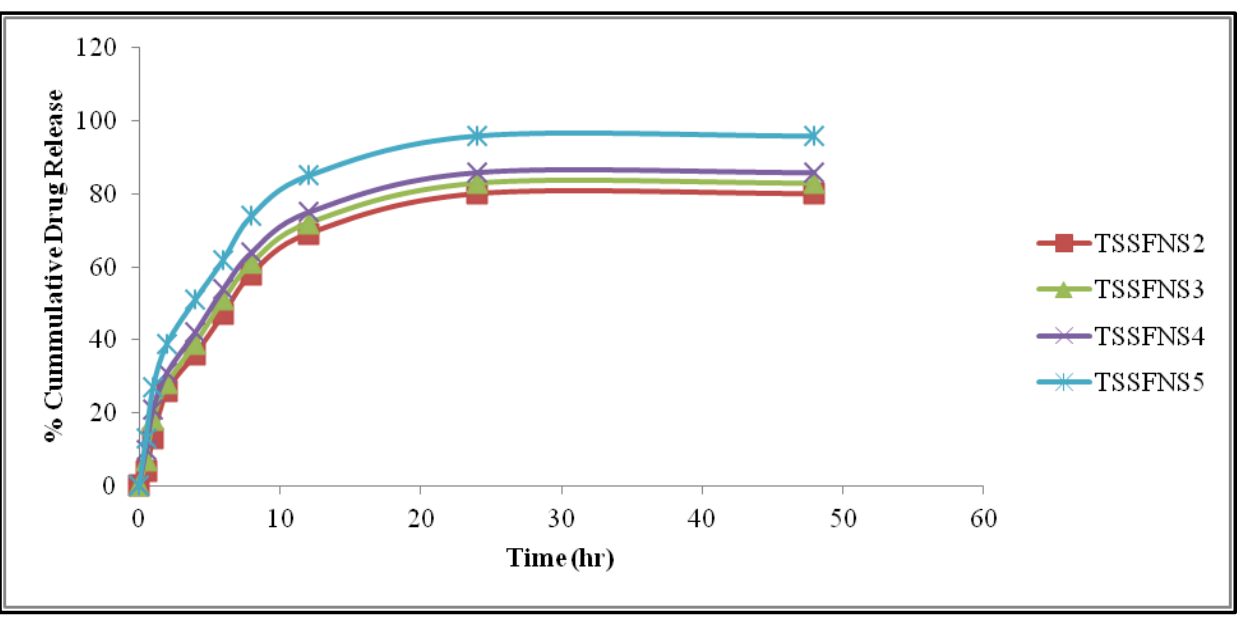

Fig. 8: \% Cumulative percentage drug release of nanospheres of telmisartan TSSF-NS2 to TSSF-NS5 (mean \pm standard deviation, $n=3$ )

Table 5: Correlation co-efficient, reaction rate-constants and diffusion-exponent of the model equations applied to the release of telmisartan from silk fibroin nanospheres

\begin{tabular}{|c|c|c|c|c|c|c|}
\hline \multirow[t]{2}{*}{ Formulation code } & Zero order & First order & \multicolumn{2}{|c|}{ Higuchi } & \multicolumn{2}{|c|}{ Korsmeyer-peppas } \\
\hline & $\mathbf{R}^{2}$ & $\mathbf{R}^{2}$ & $\mathbf{R}^{2} \mathbf{k}_{\mathrm{H}}$ & & $\mathbf{R}^{2} \mathbf{n}$ & \\
\hline TSSF-NS2 & 0.818 & 0.848 & 0.912 & 21.39 & 0.903 & 0.546 \\
\hline TSSF-NS3 & 0.838 & 0.896 & 0.934 & 23.56 & 0.919 & 0.568 \\
\hline TSSF-NS4 & 0.808 & 0.821 & 0.922 & 19.23 & 0.912 & 0.595 \\
\hline TSSF-NS5 & 0.866 & 0.979 & 0.996 & 15.31 & 0.989 & 0.622 \\
\hline
\end{tabular}

TSSF-NS= Telmisartan+Silk Fibroin Nanospheres, $\mathrm{R}^{2}$ is the coefficient of correlation, $\mathrm{k}_{\mathrm{H}}$ is the release rate constant of Higuchi model; and $\mathrm{n}$ is the release exponent of Korsemeyer-Peppas model, the release regression values (correlation coefficient values) were the greatest fitting with the Higuchi model, and the exponent values were established to lie between $(0.912-0.996)$. Therefore, the $(\mathrm{n})$ values are higher than 0.5 indicating a non Fickian release.

The in vitro cumulative drug release of silk fibroin nanosphere of telmisartan (TSSFNS1-TSSF-NS5) showed that highest $96.04 \pm 0.22 \%$ cumulative drug release of optimized TSSF-NS5 at the end of $24^{\text {th }} \mathrm{hr}$.
During in vitro drug release studies of silk fibroin loaded with telmisartan, it was found that about 74\% (fig. 8) of drug was released within $8 \mathrm{~h}$ and about $96 \%$ of drug released at the end of $24^{\text {th }} \mathrm{h}$. 
Therefore, the optimized formulation of silk fibroin nanospheres loaded with telmisartan (TSSF-NS5) was apt to release the drug over the course of time.

\section{Release Kinetics}

The in vitro release kinetics was conducted out to ascertain the release system. The release constants were measured from the slope of the suitable plots, and regression coefficient $\left(R^{2}\right)$ by linear regression analysis. The 1st-order release values for formulations were $0.821-0.979$ and $0.818-0.866$ for zero order (table 5).

\section{CONCLUSION}

The current study concludes that the nanoprecipitation method was found to be an encouraging technique for developing the dissolution of poor water soluble drug like TS. The SF loaded telmisartan nanospheres were successfully prepared by the nanoprecipitation process in acetone. The particle size increased with an increase in SF content. Polymer concentration influences drug loading and drug release from nanospheres. Generally, drug entrapment increases with increasing concentration of polymer. The $\beta$-sheet forms of SF nanosphere matrices were induced by both acetone treatment and telmisartan loading. The intermolecular interactions between SF and drug molecules also induced SF based $\beta$-sheet conformation as indicated from FTIR and DSC analysis. XRD and DSC studies indicated rebate in crystallinity, an aspect subsidizing to dissolution rate improvement of the drug. The different SF contents did not alter the design and size of SF nanospheres. The drug release rate from SF nanospheres increased as the $\beta$-sheet form of SF nanospheres was increased. It is believed that these SF nanospheres will find potential applications in drug delivery release as drug carriers, especially poor water-soluble drugs.

\section{ACKNOWLEDGMENT}

Authors thank The Principal, and The Head, Department of Pharmaceutics, JSS College of Pharmacy, Mysuru and The Management, JSS Academy of Higher Education and Research, Mysuru for their constant support and motivation.

\section{AUTHORS CONTRIBUTIONS}

All the authors have contributed equally

\section{CONFLICTS OF INTERESTS}

\section{All authors have none to declare}

\section{REFERENCES}

1. Altman GH, Diaz F, Jakuba C, Calabro T, Horan RL, Chen J, et al. Silk-based biomaterials. Biomaterials 2003;24:401-16.

2. Wenk E, Merkle HP, Meinel L. Silk fibroin as a vehicle for drug delivery applications. J Controlled Release 2011;150:128-41.

3. Shi PJ, Goh JCH. Self-assembled silk fibroin particles: tunable size and appearance. Powder Technol 2012;215:85-90.

4. Wang XQ, Yucel T, Lu Q, Hu X, Kaplan DL. Silk nanospheres and microspheres from silk/PVA blend films for drug delivery. Biomaterials 2010;31:1025-35.

5. Li X, Qin J, Ma J. Silk fibroin/poly (vinyl alcohol) blend scaffolds for controlled delivery of curcumin. Regener Biomater 2015;2:97-105.

6. Zhang YQ, Shen WD, Xiang RL, Wang W. Formation of silk fibroin nanoparticles in a water-miscible organic solvent and their characterization. J Nanopart Res 2007;9:885-900.

7. Cao Y, Liu F, Chen Y, Yu T, Lou D, Guo Y, et al. Drug release from core-shell PVA/silk fibroin nanoparticles fabricated by onestep electrospraying. Sci Reports 2017;11913:1-9.

8. Dickerson BM, Dennis BP, Tondiglia PV, Nadeau JL, Singh KM, Drummy LF, et al. 3D printing of regenerated silk fibroin and antibody-containing microstructures via multi-photon lithography. ACS Biomaterial Sci Eng 2017;9:2064-75.

9. Shahid Ud Din Wani, Gangadharappa HV. Silk fibroin based drug delivery applications: promises and challenges. Curr Drug Targets 2018;19:1177-90.

10. Yao Y, Wang W, Li M, Ren H, Caiyu C, Jialiang Wang J, et al. Curcumin exerts its antihypertensive effect by downregulating the AT1 receptor in vascular smooth muscle cells. Scientific Reports 2016;6:1-8.

11. Velasquez MT. Angiotensin II receptor blockers. A new class of antihypertensive drugs. Arch Fam Med 1996;5:351-6.

12. Dasgupta C, Zhang L. Angiotensin II receptors and drug discovery in cardiovascular disease. Drug Discovery Today 2011;16:22-34.

13. Sharma M, Sharma R, Jain KD. Nanotechnology-based approaches for enhancing oral bioavailability of poorly watersoluble antihypertensive drugs. Scientifica 2016;8525679:1-11.

14. Patela J, Dhingania A, Garala K. Design and development of solid nanoparticulate dosage forms of telmisartan for bioavailability enhancement by integration of experimental design and principal component analysis. Powder Technol 2014;258:331-43.

15. Park J, Cho W, Cha KH, Ahn J, Han K, Hwang SJ. Solubilization of the poorly water-soluble drug, telmisartan, using supercritical anti-solvent (SAS) process. Int J Pharm 2013;441:50-5.

16. Kim UJ, Park J, Kim HJ, Wada M, Kaplan DL. Three-dimensional aqueous-derived biomaterial scaffolds from silk fibroin. Biomaterial 2005;26:2775-85.

17. Hofmann S, Wong Po Foo CT, Rossetti F. Silk fibroin as an organic polymer for controlled drug delivery. J Controlled Release 2006;111:219-27.

18. Jin HJ, Park J, Karageorgiou V, Kim JU, Valluzzi R, Cebe P, et al. Water-stable silk films with reduced $\beta$-sheet content. Adv Funct Mater 2005;15:1241-7.

19. Simchua W, Narkkong NA, Baimark Y. Silk fibroin nanospheres for controlled gentamicin sulfate delivery. Res J Nanosci Nanotech 2011;1:34-41.

20. Meinel L, Hofmann S, Karageorgiou VC, Kirker Head C, McCool J, Gronowicz G, et al. The inflammatory responses to silk films in vitro and in vivo. Biomaterials 2005;26:147-55.

21. Rockwood DN, Preda RC, Yücel T, Wang X, Lovett ML. Materials fabrication from bombyx mori silk fibroin. Nat Protoc 2011;6:1612-31.

22. Chen M, Shao Z, Chen X. Paclitaxel-loaded silk fibroin nanospheres. J Biomed Mater Res Part A 2012;100A:203-10.

23. Kundu J, Chung Y II, Kim HY. Silk fibroin nanoparticles for cellular uptake and control release. Int J Pharm 2010; 388:242-50.

24. Ling SJ, Qi ZM, Knight DP, Shao Z, Chen X. Synchrotron FTIR microspectroscopy of single natural silk fibers. Biomacromolecule 2011;12:3344-9.

25. Wu M, Yang W, Chen S. Size-controllable dual drug-loaded silk fibroin nanospheres through a facile formation process. J Mater Chem B 2018;6:1179-86.

26. Costa P, Sousa LJM. Modeling and comparison of dissolution profiles. Eur J Pharm Sci 2001;13:123-33.

27. Baimark Y, Srisuwan Y. Silk fibroin spheres crosslinked by polyethylene glycol diglycidyl ether for drug delivery. Int J Chem Appl 2012;4:259-69.

28. Higuchi T. Mechanism of sustained-action medication theoretical analysis of the rate of release of solid drugs dispersed in solid matrices. J Pharm Sci 1963;52:1145-9.

29. Korsmeyer WR, Gumy R, Doelker E, Buri P, Nikolaos A Peppas. Mechanisms of solute release from porous hydrophilic polymers. Int J Pharm 1983;15:25-35.

30. Jin HJ, Park J, Karageorgiou V, Kim UJ, Valluzzi R, Cebe P, et al. Water-stable silk films with reduced $\beta$-sheet content. Adv Funct Mater 2005;15:1241-7.

31. Tanaka T, Tanigami T, Yamaura K. Phase separation structure in poly (vinyl alcohol)/silk fibroin blend films. Polym Int 1998;45:175-84.

32. Numata K, Subramanian B, Currie HA, Kaplan DL. Bioengineered silk protein-based gene delivery systems. Biomaterials 2009;30:5775-84.

33. Gupta V, Aseh A, Ríos CN, Aggarwal BB, Mathur AB. Fabrication and characterization of silk fibroin-derived curcumin nanoparticles for cancer therapy. Int J Nanomed 2009;4:115-22.

34. Yu Qing Z, Ru Li X, Hai Bo Y, Xiao Xiao C. Preparation of silk fibroin nanoparticles and their application to immobilization of L-asparaginase. Chem J Chin Univ 2008;29:628-33. 
35. Sahoo SK, Suresh P, Acharya U. Design and development of selfmicroemulsifying drug delivery systems (SMEDDS) of telmisartan for enhancement of in vitro dissolution and oral bioavailability in rabbit. Int J Appl Pharm 2018;10:117-26.
36. Hiendrawan S, Hartanti AW, Veriansyah B, Widjojokusumo E, Tjandrawinata RR. Solubility enhancement of ketoconazole via salt and cocrystal formation. Int J Pharm Pharm Sci 2015;7:160-4 